\title{
A visão do paciente atendido pelo acadêmico de medicina em um Hospital Escola
}

\author{
The perception of patients who receive care from medical students in a School Hospital
}

Luísa Lobato Macias', Renan Mota Parafita', Cezar Augusto Muniz Caldas ${ }^{1}$

\begin{abstract}
Resumo Objetivo: avaliar a opinião dos pacientes quando atendidos por acadêmicos de medicina em um Hospital Universitário. Método: trata-se de um estudo exploratório transversal, realizado por meio da utilização de questionário contendo perguntas objetivas e aplicado a pacientes do Hospital Universitário João de Barros Barreto após realizarem seus atendimentos nos ambulatórios vinculados às especialidades clínicas. Foram entrevistados 230 pacientes de agosto de 2016 a fevereiro de 2017. Foram incluídos adultos ( $\geq 18$ anos) que se encontravam em consulta médica e que concordaram em participar deste estudo através da assinatura do Termo de Consentimento Livre e Esclarecido. Foram excluídos os que não conseguissem comunicar-se

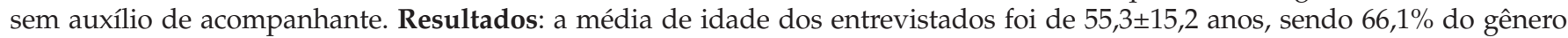
masculino. Os dados mostraram que somente 47,8\% dos entrevistados foram informados que seriam atendidos por acadêmicos; $97,8 \%$ gostaram do atendimento e $97,8 \%$ sentiram segurança na consulta. Constatou-se que a idade do paciente foi um fator relacionado sentir-se envergonhado perante o atendimento, onde, dentre aqueles com menos de 50 anos, $31 \%$ responderam que se sentiram envergonhados, enquanto os acima dessa idade, apenas $13 \%$ tiveram esta sensação ( $p=0,0030$ ). Conclusão: concluiu-se que apesar de boa parte dos pacientes não serem informados de que serão atendidos por alunos supervisionados, a maioria sente-se satisfeita e segura com o atendimento, demonstrando que não há necessidade de omitir ou receio de informar ao paciente sobre esta condição, na qual, via de regra, o paciente mostra-se satisfeito e até interessado em contribuir com a formação do estudante.
\end{abstract} Descritores: satisfação do paciente; estudantes; Hospitais Universitários; cuidados médicos.

Summary Purpose: to assess the patient's opinion when they are treated by medical students in a university hospital. Method: it consists in a cross-sectional exploratory study using a survey with objective questions answered by patients of the University Hospital João de Barros Barreto after they received medical care in the hospital clinic related to the medical specialties. From August 2016 to February 2017, 230 patients answered the survey. They were adults (18 years old or more) who were receiving medical care and agreed to participate in this study, signing a Free and Clear Term of Consent. The patients who could not communicate without the aid of an accompanying person were excluded from the study. Results: The average age of the interviewed patients was $55.3 \pm 15.2$ and $66.1 \%$ of them were men. The data showed that only $47.8 \%$ of the interviewed patients were informed that they would receive care from a medical student; $97.8 \%$ liked the medical care they received and $97.8 \%$ felt safe during the consultation. It was observed that the age of the patient was related to the feeling of embarrassment; $31 \%$ of the patients aged less than 50 years old said that they felt embarrassed in the consultation, while only $13 \%$ of the older patients had this feeling $(p=0,0030)$. Conclusion: in conclusion, even that a great number of patients are not informed that they are going to be treated by supervised medical students, most part of them fell satisfied and safe with the medical care, which shows that there is no need to omit this condition from the patient, as the patient is usually satisfied and even interested to contribute with the student's education.

Keywords: patient satisfaction; students; hospitals, university; medical care.

'Universidade Federal do Pará - UFPA, Belém, PA, Brasil

Fonte de financiamento: Nenhuma.

Conflito de interesses: Os autores declaram não haver conflitos de interesse.

Recebido: Janeiro 05, 2018

Aceito: Maio 15, 2018

Trabalho realizado no Hospital Universitário João de Barros Barreto, Universidade Federal do Pará - UFPA, Belém, PA, Brasil. 


\section{Introdução}

A interação entre estudantes e pacientes é essencial para a formação médica, e os estudantes também precisam estar cientes do valor dessa relação. Segundo estudo ${ }^{1}$ realizado com 25 estudantes do $12^{\circ}$ semestre de uma faculdade de medicina do sul do Brasil, $68 \%$ deles concordaram que o aprendizado prático ocorre durante os atendimentos, os estágios extracurriculares e no decorrer do internato, o que evidencia o reconhecimento, por parte dos pesquisados, do valor do paciente para o ensino médico.

É neste contexto de troca da relação estudante-paciente que este último vai expor sua história, seu próprio corpo, passando a ser alguém que, além de receber atendimento médico, é, também, objeto de aprendizagem prática ${ }^{2}$. Sendo assim, é importante que a relação médico-paciente seja ponderada de maneira que o paciente se sinta confortável. Considera-se que a presença dos estudantes tende a limitar a privacidade do paciente, podendo causar-lhe incomodo e constrangimento na revelação de informações, o que se confirmou em estudo realizado em $2015^{3}$, no qual 36\% dos participantes, quando questionados sobre a frequência de incômodo ou de constrangimento em razão da presença de alunos durante o atendimento, responderam "Geralmente" ou "Às vezes".

Tanto os possíveis sentimentos de insegurança, quanto o desconforto, ou, até mesmo, o constrangimento do paciente frente à presença do aluno na consulta médica, desponta a necessidade de se fornecer orientações específicas aos estudantes sobre a obrigatoriedade do respeito à vontade do paciente e da obtenção de seu consentimento, no início do atendimento, afim de serem cumpridos os princípios bioéticos da autonomia e da não maleficência. A privacidade, o bem-estar psíquico-emocional e o conforto do paciente são direitos previstos na Carta dos Direitos dos Usuários da Saúde $e^{4}$

Conhecer mais de perto este amplo espectro de percepções vivenciadas pelo paciente é de grande relevância, pois propicia ao estudante colocar, acima de tudo, a condição humana de seu ato ${ }^{5}$.

Contudo, nos dias de hoje, uma das queixas mais frequentes dos usuários e dos profissionais de saúde está relacionada com a chamada desumanização da prática médica, que pode ser entendida como uma violação do ser humano e de sua humanidade. O desconsiderar o outro, seja por ignorá-lo, seja por não valorizar suas demandas, caracteriza uma prática que merece uma análise ética. A não observância dos princípios éticos pode ser facilmente constatada nos diversos cenários de atendimento à saúde e, também, na própria escola médica, nas relações entre colegas de classe, entre os professores e seus alunos e entre médicos e pacientes. Nos últimos anos, produziu-se uma separação cada vez mais acentuada entre a ética prescrita pelos códigos e aquela que é efetivamente praticada ${ }^{6}$.

Dessa forma, é de extrema importância saber o que pensam e sentem os pacientes a respeito de seu contato com os alunos e profissionais, principalmente para a formulação das atividades práticas desenvolvidas pelas escolas médicas. Além disso, é importante, também, para aumentar os conhecimentos na área de educação médica, tendo ênfase na disciplina de Semiologia Médica e seus equivalentes, e embasar possíveis melhorias nesse ramo, fortalecendo as diretrizes de ensino, que estabelecem que o curso de graduação deve desenvolver a capacidade reflexiva e as compreensões ética, psicológica e humanística da relação médico-paciente, assim como permitir que o paciente seja tratado de forma mais ética, digna, responsável e humana e não apenas como um objeto de estudo ${ }^{7}$.

Desta forma, este estudo foi realizado para avaliar a opinião dos pacientes que são atendidos num hospital universitário sobre o atendimento realizado por estudantes de Medicina.

\section{Métodos}

Caracteriza-se por ser um estudo exploratório transversal, que foi realizado por meio da utilização de questionário contendo perguntas objetivas e aplicado a pacientes do Hospital Universitário João de Barros Barreto (HUJBB) após realizarem seus atendimentos nos ambulatórios vinculados às especialidades de clínica médica. Foram entrevistados 230 pacientes no período de agosto de 2016 a fevereiro de 2017. Este projeto recebeu parecer favorável para a sua realização pelo Comitê de Ética em Pesquisa da instituição, sob o número 1.568.180 (CAAE 56304116.7.0000.0017).

Foram incluídos adultos ( $\geq 18$ anos) que encontravam-se em consulta médica nos diversos ambulatórios das especialidades clínicas e que concordaram com a participação no presente estudo, através da assinatura do Termo de Consentimento Livre e Esclarecido (TCLE). Foram excluídos os pacientes que não conseguissem comunicar-se sem auxílio de acompanhante. 
O protocolo desenvolvido pelos próprios pesquisadores foi baseado no formulário subjetivo realizado por Gerken e colaboradores, em 2013, e utilizado no Hospital das Clínicas da Universidade Federal de Minas Gerais ${ }^{3}$. Elaborou-se, assim, um questionário fechado, o qual foi aplicado aos pacientes após os mesmos serem informados sobre os objetivos, riscos e benefícios da pesquisa, dando seu consentimento através da assinatura do TCLE.

As perguntas foram feitas baseadas em três premissas: quanto à qualidade do atendimento feito pelo aluno, se eles sabem que estão sendo atendidos em um ambiente de hospital escola e como eles se veem participando de um ambiente acadêmico. Além disso, também foram solicitadas informações gerais como sexo, idade, renda e grau de escolaridade. Em nenhum momento foi necessária a coleta de dados pessoais como nome, endereço ou número de prontuário, os quais poderiam identificar o sujeito da pesquisa.

As informações da caracterização amostral foram apuradas em banco de dados elaborado no software Microsoft ${ }^{\circledR}$ Office Excel ${ }^{\circledR}$ 2010. Na aplicação da Estatística Descritiva, foram construídas tabelas e gráficos para apresentação dos resultados e calculadas as medidas de posição como média aritmética e desvio padrão. A estatística analítica foi utilizada para avaliar os resultados das variáveis categóricas da amostra, através do Teste Qui-Quadrado e do Teste G Aderência para tabelas univariadas e Independência para cruzamentos em tabelas bivariadas. As estatísticas descritiva e analítica, foram realizadas no software BioEstat ${ }^{\circledR}$ 5.0. Para a tomada de decisão, adotou-se o nível de significância $\alpha=0,05$ ou $5 \%$, sinalizando com asterisco $\left(^{*}\right.$ ) os valores significantes.

\section{Resultados}

Foram entrevistados 230 pacientes, com média de idade de 55,3 \pm 15,2 anos, sendo $66,1 \%$ do gênero masculino e $33,9 \%$ feminino (Tabela 1 ).

Quanto aos fatores socioeconômicos, 150 (65,2\%) dos entrevistados relataram estar na faixa per capita de renda entre 1-2 salários mínimos, sendo que houve uma significância importante dos que declararam escolaridade com nível fundamental incompleto (Tabela 2).

Com relação ao tempo de acompanhamento que esses pacientes tinham no hospital, foi observada uma média de 7,9 98,9 anos, prevalecendo a faixa de 1-5 anos (Tabela 3).

Quanto aos itens relacionados à opinião dos pacientes, observou-se que 90,4\% sabiam que estavam em hospital escola, 47,8\% foram informados que seriam atendidos por acadêmicos de medicina, 97,8\% gostaram do atendimento prestado, sendo que $97,8 \%$ sentiram segurança em serem atendidos por acadêmico (Tabela 4).

Tabela 1. Gênero e faixa etárias dos pacientes entrevistados no Hospital Universitário João de Barros Barreto, no período de agosto de 2016 a fevereiro de 2017, em Belém-PA

\begin{tabular}{|c|c|c|}
\hline DADOS GERAIS & Frequência & $\%$ \\
\hline \multicolumn{3}{|l|}{ Gênero } \\
\hline Feminino & 78 & $33,9 \%$ \\
\hline Masculino* & 152 & $66,1 \%$ \\
\hline \multicolumn{3}{|l|}{ Faixa Etária (em anos) } \\
\hline$<=20$ & 5 & $2,2 \%$ \\
\hline 21 a 30 & 15 & $6,5 \%$ \\
\hline 31 a 40 & 22 & $9,6 \%$ \\
\hline 41 a 50 & 32 & $13,9 \%$ \\
\hline 51 a $60^{*}$ & 64 & $27,8 \%$ \\
\hline 61 a $70^{*}$ & 62 & $27,0 \%$ \\
\hline 71 a 80 & 22 & $9,6 \%$ \\
\hline$>80$ & 8 & $3,5 \%$ \\
\hline Média \pm DP (em anos) & \multicolumn{2}{|c|}{$55,3 \pm 15,2$} \\
\hline
\end{tabular}

${ }^{*} \mathrm{p}<0,0001$ Teste Qui-Quadrado Aderência; DP = desvio-padrão; Fonte: protocolo de pesquisa. 
Tabela 2. Dados de escolaridade e renda familiar dos pacientes entrevistados no Hospital Universitário João de Barros Barreto, no período de agosto de 2016 a fevereiro de 2017, em Belém-PA

\begin{tabular}{|c|c|c|}
\hline DADOS SOCIOECONÔMICOS & Frequência & $\%$ \\
\hline \multicolumn{3}{|l|}{ Renda familiar (Salário Minimo) } \\
\hline$<01$ & 64 & $27,8 \%$ \\
\hline 01 a $02^{*}$ & 150 & $65,2 \%$ \\
\hline$>=03$ & 16 & $7,0 \%$ \\
\hline \multicolumn{3}{|l|}{ Escolaridade } \\
\hline Fundamental incompleto* & 84 & $36,5 \%$ \\
\hline Fundamental completo & 37 & $16,1 \%$ \\
\hline Médio incompleto & 27 & $11,7 \%$ \\
\hline Médio completo & 63 & $27,4 \%$ \\
\hline Superior incompleto & 9 & $3,9 \%$ \\
\hline Superior completo & 10 & $4,3 \%$ \\
\hline
\end{tabular}

${ }^{*} \mathrm{p}<0,0001$ Teste Qui-Quadrado Aderência; Fonte: protocolo de pesquisa.

Tabela 3. Tempo de acompanhamentos hospitalar dos pacientes entrevistados no Hospital Universitário João de Barros Barreto, no período de agosto de 2016 a fevereiro de 2017, em Belém-PA

\begin{tabular}{|c|c|c|}
\hline $\begin{array}{c}\text { TEMPO QUE REALIZA } \\
\text { CONSULTAS NO HOSPITAL } \\
\text { (em anos) }\end{array}$ & Frequência & $\%$ \\
\hline$<01$ & 37 & $16,1 \%$ \\
\hline 01 a 05 & 77 & $33,5 \%$ \\
\hline 06 a 10 & 62 & $27,0 \%$ \\
\hline 11 a 15 & 26 & $11,3 \%$ \\
\hline 16 a 20 & 16 & $7,0 \%$ \\
\hline$>20$ & 12 & $5,2 \%$ \\
\hline TOTAL & 230 & $100,0 \%$ \\
\hline Média \pm DP (em anos) & \multicolumn{2}{|c|}{$7,9 \pm 8,9$} \\
\hline
\end{tabular}

$\mathrm{DP}=$ desvio-padrão; Fonte: protocolo de pesquisa.

Visando identificar algum fator que pudesse influenciar nas respostas, foram feitos cruzamentos dos dados com relação à idade, escolaridade e renda, a pergunta número 1 (referente se sabia ou não que estava sendo atendido em um hospital universitário) e a pergunta número 2 (se ele foi informado que seria atendido por acadêmico) com as perguntas de 3 a 10. O único cruzamento relevante estatisticamente foi a relação idade com o item que tratava da vergonha do paciente ao ser atendido por muitos acadêmicos, notando-se que os pacientes com idade abaixo de 50 anos responderam em 31\% das vezes que sim, sentiam-se envergonhados, enquanto os acima dessa idade apenas em $13 \%(p=0,0030)$.

\section{Discussão}

Este estudo demonstrou que a maioria dos pacientes reconheceu estar sendo atendido num hospital escola, embora a maioria não soubesse que seria atendida por um estudante. A despeito desta condição, nota-se boa relação estudante-paciente, caracterizada pela maioria ter manifestado que gostou do atendimento prestado e se sentiu seguro com o mesmo. Ainda mais, a opinião do paciente foi favorável ao atendimento prestado pelos estudantes, se sentindo como integrante do processo de formação do estudante, visto que a maioria considerou importante o atendimento realizado pelo aprendiz para a sua formação e tendo a percepção de atendimentos realizados de maneira mais atenciosa. 
Tabela 4. Resumo dos questionamentos feitos aos pacientes entrevistados no Hospital Universitário João de Barros Barreto, no período de agosto de 2016 a fevereiro de 2017, em Belém-PA

\begin{tabular}{|c|c|c|c|}
\hline Visão do paciente atendido pelo acadêmico de medicina. & $\mathbf{n}$ & $\%$ & p-valor \\
\hline Sabe que está em um hospital universitário? & 208 & $90,4 \%$ & $<0,0001$ \\
\hline $\begin{array}{l}\text { Foi informado que seria atendido por um acadêmico de medicina } \\
\text { supervisionado pelo professor médico? }\end{array}$ & 110 & $47,8 \%$ & 0,5529 \\
\hline Gostou desse atendimento? & 225 & $97,8 \%$ & $<0,0001^{*}$ \\
\hline Se sente seguro(a) sendo primeiramente avaliada por um acadêmico? & 225 & $97,8 \%$ & $<0,0001^{*}$ \\
\hline $\begin{array}{l}\text { Acha importante esse atendimento feito pelo acadêmico para o } \\
\text { aprendizado do mesmo? }\end{array}$ & 229 & $99,6 \%$ & $<0,0001^{*}$ \\
\hline Acha que o atendimento e os tratamentos são feitos com maior atenção? & 226 & $98,3 \%$ & $<0,0001^{*}$ \\
\hline Sente-se envergonhado quando atendidos por muitos estudantes? & 44 & $19,1 \%$ & 0,4995 \\
\hline $\begin{array}{l}\text { Sente-se ajudando na formação médica e contribuindo para o melhor } \\
\text { aprendizado do acadêmico? }\end{array}$ & 229 & $99,6 \%$ & $<0,0001^{*}$ \\
\hline Sente alguma frustação por ser atendido(a) por acadêmicos? & 16 & $7,0 \%$ & $<0,0001^{*}$ \\
\hline
\end{tabular}

Fonte: protocolo de pesquisa.

O presente trabalho buscou compreender como o paciente observa o processo ensino-aprendizagem dentro do hospital, até que ponto as atividades curriculares e a dinâmica diferenciada de um hospital, que apesar de ser prestador de serviço de saúde à população, o faz sob o ponto de vista academicista, o qual pode afetar a perspectiva assistencial que o paciente busca encontrar.

Inicialmente, nota-se que a população que utiliza o sistema único no HUJBB é muito carente e dotada de um perfil socioeconômico muito vulnerável, observação essa que foi confirmada pelos resultados que constatam que $65,2 \%$ dos pacientes recebiam entre 1-2 salários mínimos per capita, e com predomínio da formação apenas com ensino fundamental incompleto. Esses dados são corroborados com a literatura, onde segundo estudo publicado em $2015^{8}$, em que foram entrevistados 200 pacientes, relatou-se que $44 \%$ desses tinha o ensino fundamental incompleto e, em outro publicação ${ }^{9}$ de 2007, foi referido que usuários do Sistema Único de Saúde (SUS) são em grande parte de baixo nível socioeconômico.

A formação médica no Brasil tem como modelo um curso teórico-prático com seis anos de duração, no qual o estudante de medicina é inserido no atendimento ambulatorial e hospitalar, sendo isso um fator fundamental no seu aprendizado. Apesar disso, o papel do estudante no Hospital Universitário é pouco difundido, o que foi demonstrado em um dos maiores hospitais-escola do País, onde 36\% dos pacientes questionados não sabiam que seriam atendidos por acadêmicos ${ }^{3}$. Tal desinformação também ficou evidente no presente levantamento, no qual 52,2\% dos entrevistados não sabia que seria, inicialmente, atendido por acadêmicos no ambulatório.

Situação semelhante foi evidenciada por Geraldo Junior em 2014, da Universidade de Fortaleza, no Hospital Geral Dr. Waldemar de Alcântara, onde mostrou-se que apenas 9\% dos pacientes sabiam que estavam em um atendimento universitário ${ }^{6}$. Essa situação torna-se ainda mais grave quando, dentro do consultório, aluno e professor não informam o paciente da participação do estudante, o que contraria o Código de Ética Médica, no artigo $110^{10}$.

Tal desencontro de informações a respeito da participação do aluno e do acompanhamento do professor durante a consulta no Hospital Universitário se propaga para o interior do consultório, quando tutor e aluno consideram desnecessário informar o modelo de consulta ao paciente ${ }^{11}$. Isto se deve por ambos partirem da premissa de que, por se tratar de um Hospital Universitário, os atendimentos serão realizados por estudantes supervisionados. Com isso, encontra-se uma discrepância entre a resposta ao quesito "sabe que está em um hospital universitário?" e "foi informado que seria atendido por um acadêmico de medicina supervisionado pelo professor médico?".

Evidencia-se, ainda, que não há uma preocupação institucional em prestar essa informação, uma vez que apenas, $6,9 \%$ dos pacientes entrevistados no estudo feito por Gerken et al. ${ }^{3}$ foram informados da atividade docente-assistencial no momento da marcação da consulta. 
Apesar de mais da metade não ser informada que seria atendido por estudantes em formação, $97,8 \%$ relataram que gostaram do atendimento prestado e que se sentiram seguros com o atendimento. Esse mesmo perfil foi referido em dados obtidos de uma análise ${ }^{3}$ de 2013 a qual mostrou que, apesar da falta de informação sobre o atendimento inicial feito por acadêmico, o paciente costuma se sentir bem com essa abordagem inicial, com dados subjetivos de que teriam o seu diagnóstico mais discutido e que os alunos costumam ser bem atenciosos e curiosos. Desta forma, não parece ser justificável ou mesmo razoável a necessidade de omitir a natureza do atendimento, inicialmente por um estudante, para o paciente, para que o mesmo esteja pleno das informações que têm direito e que o estudante possa familiarizar-se com uma troca de informações com o paciente baseada na honestidade e no respeito à ética.

$\mathrm{Na}$ análise deste estudo, destaca-se avaliar a participação dos pacientes quanto ao processo de ensinoaprendizagem do estudante, se eles se sentiam contribuindo ou não no processo de formação. Tal circunstância pode ser confirmada com $99,6 \%$ dos participantes relatando que acha importante essa forma de atendimento para o aprendizado do acadêmico e que se sentia ajudando na formação deste. Essa afirmativa também foi confirmada por pesquisa ${ }^{6}$, datada de 2015, na qual $98 \%$ da amostra sentiu-se satisfeita por contribuir para o aprendizado do estudante de medicina, assim como na análise ${ }^{12}$, feita em 2010, com estudantes em unidades básicas de saúde, quando a população foi questionada se a presença dos estudantes naquela região poderia melhorar o atendimento, na qual $98 \%$ responderam que sim. Apesar desta última abordagem não ter sido feita num hospital escola como adotado nesta pesquisa, é mais um resultado satisfatório que corrobora, positivamente, para a presença do aluno e, consequentemente, do curso de Medicina dentro de ambientes assistenciais.

Tendo em mente o interesse em entender as variáveis que poderiam interferir nesta relação estudantepaciente, o estudo presente resultou em análises bivariadas que constataram que pacientes acima dos 50 anos, quando comparados com os abaixo dessa faixa etária, sentiam-se menos envergonhados ao serem atendidos por acadêmicos. Isto contraria o dado encontrado na pesquisa feita por Berwanger et al..$^{8} \mathrm{em} 2015$, na qual pacientes jovens, bem informados e com poucas comorbidades tenderam a sentirem-se menos constrangidos. Por outro lado, no mesmo estudo, a população idosa, com menos escolaridade e inúmeras comorbidades, tendeu a ser menos receptiva às consultas com acadêmicos presentes. Acredita-se que tal resultado deve-se ao fato de que a amostra analisada apresentou maior número de participantes na faixa etária acima de 50 anos e ensino fundamental incompleto, inferindo-se que esse aspecto possa ter contribuído para que uma parcela relativamente importante referisse incômodo ou constrangimento.

\section{Conclusões}

Este estudo destaca uma boa relação entre os estudantes e os pacientes analisados, embora muitos não tenham sido esclarecidos sobre o atendimento inicial realizado por estudantes.

As opiniões, no geral, positivas dos pacientes com relação ao atendimento realizado pelos estudantes, demonstra que não há necessidade de omitir ou receio de informar ao paciente sobre esta condição, na qual, via de regra, o paciente mostra-se satisfeito e, até, interessado em contribuir com a formação do estudante.

Atenção especial deve ser prestada aos pacientes mais jovens, que, de acordo com os dados apresentados, sentem-se mais envergonhados ao ser atendidos por estudantes. Tal situação demonstra a necessidade de esclarecimento aos pacientes e de respeito aos seus sentimentos, visando uma relação estudante-paciente e, futuramente, uma relação médico-paciente, baseada em princípios éticos e em confiança mútua.

\section{Referências}

1. Grosseman SSC, Stoll C. O ensino-aprendizagem da relação médico-paciente: estudo de caso com estudantes do último semestre do curso de medicina. Rev Bras Educ Med. 2008;32(3):301-8. http://dx.doi.org/10.1590/S0100-55022008000300004.

2. Menéndez DFS, Azevedo MH, Paiva AFA, Sousa RL, Silva IBA, Maroja JLS. Percepção do paciente na interação com o aluno de semiologia médica: Proposta para questionário estruturado de observação. In: Anais do 10 Encontro de Iniciação à Docência; 2008 [citado em 13 abr 2017]. Disponível em: http://www.prac.ufpb.br/anais/IXEnex/iniciacao/documentos/anais/6.SAUDE/6CCSDMIMT09.pdf

3. Gerken IB, Andrade CS, Lopes FB, Ribeiro MMF. Conhecimento e Opinião de Pacientes sobre Prática Docente-Assistencial. Rev Bras Educ Med. 2013;37(1):66-71. http://dx.doi.org/10.1590/S0100-55022013000100010.

4. Brasil. Ministério da Saúde (BR) [Internet]. Carta dos Direitos dos Usuários da Saúde. Brasília; 2011 [citado em 13 abr 2017]. Disponível em: use.ufscar.br 
5. Jardim PCBV, Costa NGS, Oliveira PC, Silva VN, Rabelo YS. O papel do aluno de graduação em Medicina no atendimento a pacientes de enfermarias de longa permanência de um hospital-escola. Rev Bras Educ Med. 2008;32(1):75-82. http://dx.doi.org/10.1590/ S0100-55022008000100010.

6. Taquette SR, Rego S, Schramm FR, Soares LL, Carvalho SV. Situações eticamente conflituosas vivenciadas por estudantes de medicina. Rev Assoc Med Bras. 2005;51(1):23-8. http://dx.doi.org/10.1590/S0104-42302005000100015. PMid:15776181.

7. Silva GB Jr, Duarte RP, Menezes AR, Morais AP, Alves AM, Daher EF. Percepção dos pacientes sobre aulas práticas de medicina: uma outra ausculta. Rev Bras Educ Med. 2014;38(3):381-7. http://dx.doi.org/10.1590/S0100-55022014000300013.

8. Berwanger J, Geroni GD, Bonamigo EL. Estudantes de medicina na percepção dos pacientes. Rev Bioét. 2015;23(3):552-62. http://dx.doi. org/10.1590/1983-80422015233092.

9. Paiva SMA, Gomes ELR. Assistência Hospitalar: Avaliação Da Satisfação Dos Usuários Durante Seu Período De Internação. Rev Latino-am Enfermagem. 2007;15(5):1-7.

10. Conselho Federal de Medicina (BR) [Internet]. Código de Ética Médica. 2009 [citado em 13 abr 2017]. Disponível em: http://www. portalmedico.org.br/resolucoes/cfm/2009/1931_2009.htm

11. Thomaz AKB. O ensino-assistência nas disciplinas Semiologia I - Módulo Adultos, Medicina Geral de Adultos I e Doenças Infecciosas e Parasitárias da Faculdade de Medicina da UFMG, praticado nos Ambulatórios do Hospital das Clinicas da UFMG: o olhar do cliente [dissertação]. Belo Horizonte (MG): Faculdade de Medicina, Universidade Federal de Minas Gerais; 2008.

12. Almeida FCM, Maciel APP, Bastos AR, Barros FC, Ibiapina JR, Souza SMF, et al. Avaliação da inserção do estudante na unidade básica de saúde: visão do usuário. Rev Bras Educ Med. 2012;36(1):33-9. http://dx.doi.org/10.1590/S0100-55022012000200005.

\section{Autor correspondente}

Cezar Augusto Muniz Caldas

Av. Tavares Bastos, 1485, Bloco 02, Ap. 304, Marambaia

CEP 66615-005, Belém, PA, Brasil

Telefone.: (91) 3231-0359 / 99915-7041

E-mail: cezarcaldas@ufpa.br

\section{Informação sobre os autores}

LLM e RMP são graduados em Medicina pela Universidade Federal do Pará (UFPA).

CAMC é doutor em Ciências Médicas pela Faculdade de Medicina da Universidade de São Paulo (FMUSP); docente do Curso de Medicina da Universidade Federal do Pará (UFPA)

\section{Contribuição dos autores}

LLM, RMP e CAMC foram responsáveis pelo desenho do estudo, análise dos dados, elaboração no manuscrito e revisão final. LLM e RMP também coletaram os dados. CAMC também foi responsável pela orientação na execução das atividades.

Todos os autores leram e aprovaram a versão final submetida ao Pará Research Medical Journal. 